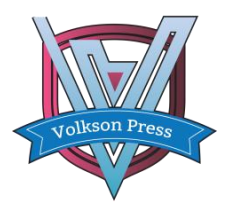

Contents List available at VOLKSON PRESS

Computer Science and Artificial Intelligence(CSAI)

DOI : http://doi.org/10.26480/iscsai.01.2017.29.32

\title{
A Novel Intelligent Light Nurturing System
}

\author{
Hong Zhou ${ }^{\mathrm{a}}$, Xiaolei Shen ${ }^{\mathrm{b}}$, Jiachi Zhang ${ }^{\mathrm{c}}$ and Wei Shu ${ }^{\mathrm{d}}$ \\ a,b,c,dDepartment of Instrument Science and Engineering, Zhejiang University, Hangzhou, China \\ ae-mail: zhouh@mail.bme.zju.edu.cn \\ ce-mail: zhangiiachi1007@gmail.com \\ *Corresponding Author: zju.shen2017@gmail.com
}

This is an open access article distributed under the Creative Commons Attribution License, which permits unrestricted use, distribution, and reproduction in any medium, provided the original work is properly cited

\section{ARTICLE DETAILS}

\section{Article History:}

Received 02 october 2017

Accepted 06 october 2017

Available online 11 october 2017

Keywords:

light nurturing system, LED,

embedded database, fuzzy

control algorithm

\section{ABSTRACT}

In this paper, we design an intelligent light nurturing system which assists researches in performing light nurturing experiments. The main controller of system controls 1 to 3 incubators. The main controller selects S5PV210 as the core hardware platform, selects Linux and QT as the software platform. And the system integrates SQLite database and Apache web server. In the incubator, we use the embedded system with the LPC2292 MCU to communicate with the main system, control the detection of environmental parameters, control LED lights, acquire images, etc. And we use fuzzy control algorithm to achieve temperature and humidity control in the incubator. The experimental results show that the designed system is reliable and stable. The control effect of temperature and humidity is robust, and the system well assists researchers in light nurturing experiments.

\section{Introduction}

Plant photosynthesis is the basis of all life on the earth. Photosynthesis of plants and algae is affected by the light intensity, spectrum and photoperiod. Generally, the light incubator provides artificial light sources and controls the light intensity, spectrum and photoperiod. In plant germination experiments, the system can be used to research the impact of photosynthesis of plants and algae in light intensity, spectrum and photoperiod. And then, it provides theoretical bases for seed germination, plant breeding and production and the cultivation of algae. It is the ideal test equipment for biogenetic engineering, medicine, agriculture, forestry, environmental science, animal husbandry, aquatic products and scientific research departments.

At present, most of the light nurturing equipment mainly uses incandescent lamp, fluorescent lamp, high pressure mercury lamp as artificial light. These only can study the influence of light period on the growth of the plant, but the spectrum and light intensity not ideal enough to meet most experimental requirements. Meanwhile, the luminous efficiency, the lamp heat and the lamp life of the artificial light are not ideal. And the parameters can't be controlled. The nurturing system equipped with multiple wavelength LED can set different spectra and color of light for the plant growth environment. Compared with the system equipped with traditional artificial light source, the system equipped with LEDs has the advantages of low energy consumption, low cost, adjustable light intensity and spectrum, low cooling load, high electro-optical conversion efficiency, small size and long service life. LED light source is a very suitable artificial light source for plant culture experiment $[1,2]$.

Compared with the foreign light nurturing equipment, the domestic equipment has many shortcomings of simple functions, simple control system, rough user interface. The domestic equipment only provides the control of the light cycle, but the setting of light color, temperature, humidity, collection and control functions can't be completed. So many defects led to the domestic equipment can't complete the complex experiments. Therefore, this paper designs an intelligent lighting nurturing system based on LED, and the system uses the fuzzy control algorithm to control temperature and humidity. And we design a new user interface to assist researchers perform light nurturing experiments [3].

\subsection{SYSTEM DESIGN}

The multifunctional light nurturing system can control one or more light incubators parameters such as light color, light intensity and light period. And the system can monitor and control parameters such as temperature, humidity and $\mathrm{CO} 2$ concentration in the incubator at the same time. The system has good functions of experimental design and record. And users can design complex experiment task, record and upload the experiment data. The system has long-range communication ability. The user can monitor the experiment through the network, needn't operate the experiment on the scene.

\subsection{System control scheme}

Traditional light incubator is generally a single structure, that is a controller control one incubator. But this will waste system resources and increase costs. The system we designed uses a master-slave structure. The minimum system is a main incubator, and the largest system are a main incubator and two subordinate incubators. The system uses RS485 protocol to communicate with incubators and control them. A main incubator includes control panel, human-computer interaction board and driver board for acquisition. A subordinate incubator includes drive board acquisition and no-controlling unit. Therefore, A subordinate incubator can't work independently. The host system consists of the main control unit (MCU) Samsung S5PV210 of CortexTM-A8, display control unit, communication unit. The MCU of incubator

ARM7 processor LPC2292. The overall structure shown in Figure 1.

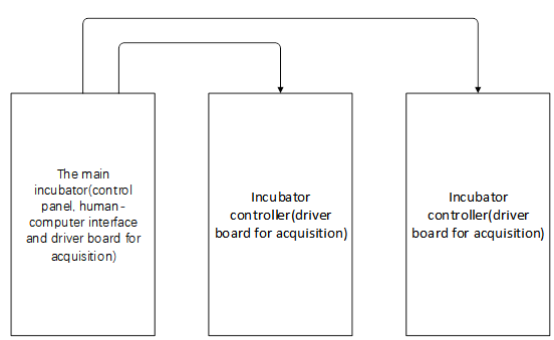

Figure 1: The overall structure of system 
The traditional intelligent light incubator generally uses the keyboard to setting and digital tube to display. The display for the task is not clear, so the users are prone to make mistakes in the operation process and lead to the experiment failure. The system human-computer interface is designed through QTGui. The experiment task and parameters are shown by pictures, tables and text. Users can use the touch screen or USB wireless controller to operate the system directly. Through the system prompts and simple operation, we can complete the parameter settings of experiment.

\section{$1.4 \quad$ Multitasking experiment design}

The user can set up 10 different experiments for each incubator and each experiment can be divided into 40 tasks. Each task can set several parameters such as light cycle, light intensity, light color, temperature, humidity, $\mathrm{CO} 2$ concentration, etc. to meet the requirements of complex experiments.

\subsection{Experiment data recording and storage}

Experiment data recording is an important part of the experiment of light nurturing. The experiment data of the traditional light incubator need to be manually recorded by the experimenter. The system can automatically record and store all the information of experiment operation and incubator system within one month. And users can set the image recording function the system takes photos of the internal incubator through the camera. Files can be stored in the system or SD of user and be uploaded to the server through the network. It's convenient for users to analyze the experiment data.

\subsection{System security}

In general, the traditional lighting incubators do not consider the design of system security. Anyone can operate the incubator lead to experiment face safety risks. For the experiment data security, the system needs to log in before experiment setup and system operation. There are two ways to log in, one is user name and password, the another is fingerprint. The system can store 20 password users and 20 fingerprint users at the same time. After logging in the system, the setting of experiment will be automatically marked by the user's system and be stored in the lab record.

1.7

Remote control

Currently, the light incubators do not have the remote controlling function. With the development of network technology and embedded technology, remote controlling system has become an important part of advanced embedded systems. The user can view the current experiment data and task status through the webpage. At the same time, the user can view the screen of system camera monitoring through the webpage and view the real-time image information inside the incubator.

1.8

LED multi-color spectrum controlling

The intelligent controlled LED cold light is as the incubator light source. According to demand, users can choose LED light color, adjust the spectrum, set specific experiments.

\section{SYSTEM SOFTWARE DESIGN}

The system provides many functions including the user human-computer interface, web server, SMS control services, embedded database. Incubator controller includes timely response to the main control system commands, control incubator hardware and real-time feedback of cabinets environment information. The application software mainly includes three parts shown in Figure 2.

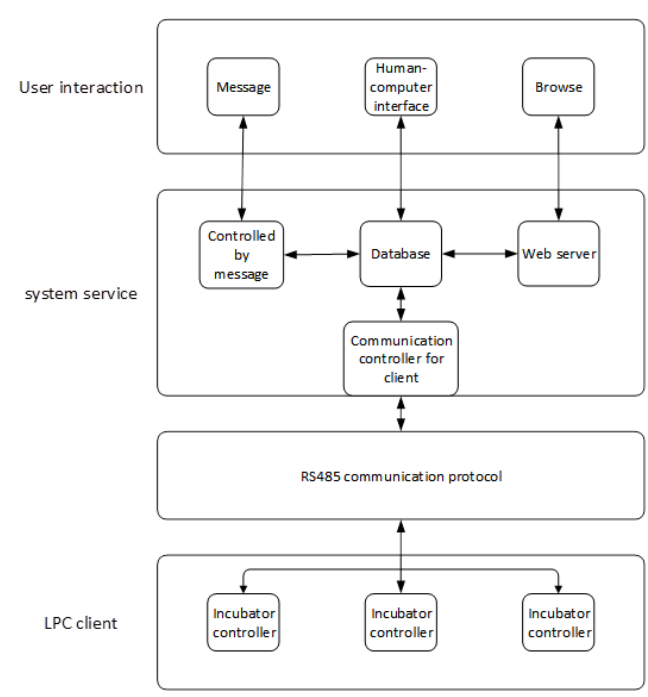

Figure 2: The structure of application software

$2.1 \quad$ User interface part

We carefully designed the User Interface (UI) for users' interaction with the system and the system's easily parsing user commands. Users can browse and supervise the system through three main access:

\subsubsection{Message}

Users can request the current status information of the system. Specific commands are required and responds including status information, such as current temperature, humidity and illumination intensity, are guaranteed to return. Users can also enquiry experimental information, current tasks' parameters, etc.

\subsubsection{Browser}

We deplored Apache Server at the backend of the system so that users can acquire system information through browsers like visiting a website. The function of supervising the system is mainly achieved by php scripts. Users can not only enquiry system information but can also inspect current status through web camera. The remote video monitor can transmit image information of incubators so that the users will have a real-time observation.

\subsubsection{Direct UI}

Despite the remote controlling UI, we also provide direct UI and this is the main approach for users' interaction. Users need to provide their username and password, or they can also login by fingerprint identification. After login, users can supervise every incubator, set system experiments and tasks, change system parameters and internet settings. Users can take advantage of every function of the system through this interface.

\section{$2.2 \quad$ System Service Part}

The system service part is mainly responsible for parsing users' commands, supervising and controlling lower machines. This part consists of several components below.

\subsubsection{Embedded database}

We adopt the embedded database SQLite to store system information. The information comprises of experiment information, task information, system setting information and user information. The database involves in almost every process of the system, such as in order to display the status information we have to extract the information from the database or by adding and revising the records in the database, we can reset the status of the system.

\subsubsection{Message Controller}

The message controller will monitor users' message requests. After receiving the massage, the controller will give corresponding operations to the database and send a message back to the users according to the commands in users' messages 
We adopt Apache Server and php dynamic scripts to respond to browser users' requests. Users can choose experiment indexes to acquire correlated experiment and task information. Users can also visit the camera webpage to inspect the incubator through video stream.

\subsubsection{Client Communication Controller}

The controller is a bidirectional information transmitter between upper computers and lower machines. On the one hand, the controller can read the information in the database, wrap the controlling information and send the package to lower machines. On the other hand, the controller can also unpack packages from lower machines, extract and category useful information then save them in the database.

\section{$2.3 \quad$ LPC Client}

The client is embedded in the incubator and will respond to upper computers' requests, parse the controlling commands, read command information and control the hardware in the incubators to change the environment conditions like lighting, temperature and humidity.

\subsubsection{Temperature and Humidity Fuzzy Control Algorithm}

The system is equipped with temperature and humidity sensor to detect temperature and humidity information independently. According to the pragmatic experiments, we found that the traditional control algorithm faced several problems. Then we adopted a fuzzy control algorithm which improve the system's precision and stability $[4,5]$. The model of temperature and humidity is close to first-order lag system. As presented below, temperature's lag time is longer than humidity's.

$$
T(s)=\frac{K e^{-\tau s}}{T S+1}
$$

$\mathrm{K}$ is the system's gain factor and T is the time constant. Due to the coupling phenomenon as manifested in figure 3 , temperature and humidity will change as the other changes. These two physical quantities are closely coupled and are highly influenced by the environment. When we need to adjust system's temperature or humidity, the holistic environment will cost relatively too much time to reach the proper condition because of the control model. It is nearly impossible to obtain a high increase speed, short transition time and a small overshoot with traditional control algorithms.

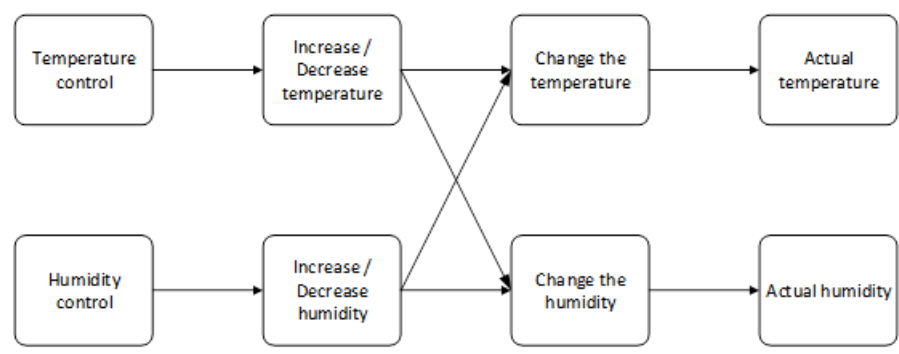

Figure 3: Temperature and humidity coupling phenomenon

When we heat the system and the temperature increase, water will be vapored so the humidity increase. However, when we want to decrease the temperature, vapor will be cooled to condense then the humidity drops.

Taking the advantage of incubators' similar specification and uniform components, we adopt a fuzzy control algorithm basing on an experiential model [6]. The model is different from math model and is totally designed by experiments records in our nurturing environments. We store the records in a table and every time we want to change the environments, the system will adjust quickly and effectively according to the table.

The incubators controller can be divided into two parts, temperature controller and humidity controller. We divide temperature scale into 9 levels and every level is one temperature unit higher or lower than its contiguous levels. The temperature control table and humidity control table are respectively demonstrated as table 1 and table 2 below. According to the tables, we can rebuild the fuzzy control model as demonstrated in figure 4.

Table 1: Temperature control table. We can take the first row as an example, when we the current temperature is 4 temperature units lower than the target condition, we have to increase 5 temperature units and increase 4 humidity units instead of directly increase 4 temperature units. Temperature Theoretical temperature Actual temperature Humidity \begin{tabular}{lll} 
difference & compensating & compensating \\
\hline
\end{tabular}

\begin{tabular}{llll}
\hline-4 & & & \\
-3 & 4 & 5 & 3 \\
-2 & 3 & 4 & 1 \\
-1 & 2 & 2 & 0 \\
0 & 1 & 1 & 0 \\
1 & 0 & 0 & 0 \\
2 & -1 & -1 & -1 \\
3 & -2 & -3 & -3 \\
4 & -3 & -4 & -4 \\
\hline
\end{tabular}

Table 2: Humidity control table. The control method is similar to Table 1.

\begin{tabular}{|c|c|c|c|}
\hline $\begin{array}{l}\text { Humidity } \\
\text { difference }\end{array}$ & $\begin{array}{ll}\begin{array}{l}\text { Theoretical } \\
\text { compensating }\end{array} & \text { humidity } \\
\end{array}$ & $\begin{array}{l}\text { Actual humidity } \\
\text { compensating }\end{array}$ & Temperature \\
\hline-4 & 4 & 5 & -4 \\
\hline-3 & 3 & 4 & -3 \\
\hline-2 & 2 & 2 & -1 \\
\hline-1 & 1 & 1 & 0 \\
\hline 0 & 0 & 0 & 0 \\
\hline 1 & -1 & -1 & 0 \\
\hline 2 & -2 & -3 & 1 \\
\hline 3 & -3 & -4 & 3 \\
\hline 4 & -4 & -5 & 4 \\
\hline
\end{tabular}

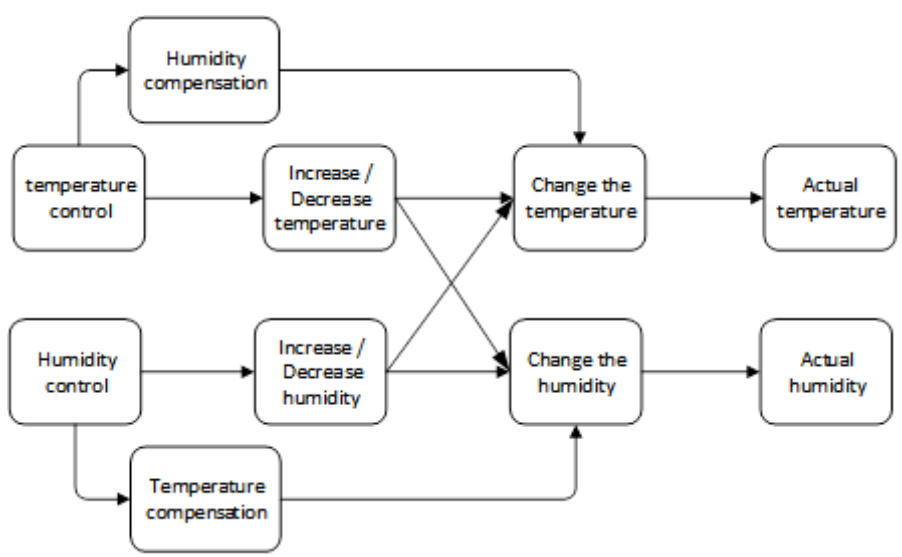

Figure 4: Temperature and Humidity Fuzzy Control Model

\section{SUMMARY}

In this nurturing system, we select Linux as the operating platform and QtGui as the interface for users to interact with the system. Compared with traditional control system, our system is more user-friendly and more convenient to operate. Besides, traditional control system of similar type is usually isolated. Although distributed, every incubator in our system is connected to a server then a bidirectional communication channel is built. Users can control the system through remote approach and acquire valuable information saved in the server. In this way, the experiments will be well supervised and adjusted. Practical application has proved the system reliable and stable enough to be a competent auxiliary for light nurturing experiments. We are looking forward to its further contribution to development of intelligent light nurturing system.

\section{ACKNOWLEDGMENT}

This work is supported by National 863 Project named "LED energysaving light source and Intelligent control technology of luminous environment for plant factory" (item number: 2013AA103001).

\section{REFERENCE}

[1] Lv, Y.J., Li, S.G., Zhou, X.H. 2003. Research Progress of Ornamental Orchid Plants In Tissue Culture And Genetic Transformation. China Biotechnology, 23 (10), 42-46. Doi: 10.3969/J.Issn.16718135.2003.10.009.

[2] Liu, Y.T., Wang, R.G. 2008. New Trends of LED Application. Ome Information, 25 (10), 30-34. Doi: 10.3969/J.Issn.1007-1180.2008.10.007.

[3] Zhong, Y.S., Yang, J.Q., Deng, J.L. 2001. Multivariable Fuzzy Control of Temperature and Humidity in A Greenhouse. Transactions of The Chinese Society of Agricultural Machinery, 32 (3). Doi: 10.3969/J.Issn.10001298.2001.03.022.

[4] Posthoff, C., Sonntag, P. 1994. Fussy Methods in Expert Systems for Configuration and Control (Models, Implementations and Experiences). Fuzzy Systems, 1994. IEEE World Congress on Computational Intelligence. Proceedings of The Third IEEE Conference On, 1 (1), 337-339. IEEE. 
[5] Fogarty, T.C. 1993. Classifier Systems for Control. Genetic Algorithms For Control Systems Engineering, IEE Colloquium On (Pp.8/1-8/3). IET.
[6] Wei, S. 2013. Intelligent Light Nurturing System. Doctoral Dissertation, Zhejiang University.

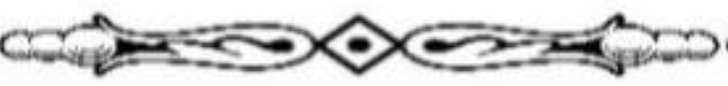

